\title{
Comparación de la eficiencia de trampas Barber y Malaise para el estudio de la biodiversidad de Hexapoda de vegas altoandinas
}

\author{
Comparison of efficiency of Barber and Malaise traps to study \\ the biodiversity of Hexapoda of Andean peatlands \\ Jorge Cepeda-Pizarro ${ }^{*}$, Christian González A. ${ }^{2}$, Carlos Zuleta R. $^{3}$, Jaime Pizarro-Araya ${ }^{1}$
}

\section{RESUMEN}

Se reporta la aplicabilidad de dos métodos de captura y muestreo de la fauna de Hexapoda asociada a vegas andinas. En el presente estudio se usaron los diseños Barber y Malaise, los que fueron instalados en tres vegas localizadas en una subcuenca altoandina de la hoya hidrográfica del río Elqui (Región de Coquimbo, Chile). El estudio duró 3 meses y se realizó en la estación de mayor actividad biológica. En el análisis de la riqueza taxonómica (Orden para Hexapoda y Familia para Diptera) se usaron los estimadores no paramétricos Chao-1, Chao-2, Jack-1, Jack-2 y Boostrap. En conjunto y por todo el estudio se capturaron 88217 especímenes; registrándose una riqueza de 14 órdenes de Hexapoda y 29 Familias de Diptera. Tanto cualitativa como cuantitativamente las trampas difirieron en el registro de los taxa presentes. A nivel ordinal, el muestreo combinado entregó una representación del 99\% de los órdenes de Hexapoda presentes en las vegas. En el caso del trampeo Barber, esta representación alcanzó el 98\%; mientras que bordeó el $62 \%$ con las trampas Malaise. A nivel de Familias de Diptera, el muestreo combinado entregó una representación del $\sim 98 \%$. Con el trampeo Barber esta fue $\sim 69 \%$, mientras que bordeó el $84 \%$ con el trampeo Malaise. Aunque la trampa Malaise fue más afectada por las condiciones ventosas que la trampa Barber, la primera actuó como un buen complemento de la segunda, considerando que esta no solo capturó especímenes ligados al suelo, sino también capturó una fracción importante de hexápodos asociados al follaje y voladores. De los resultados de este trabajo se desprende que la trampa Barber es un artificio adecuado para estudios de la fauna de hexápodos de estos ambientes. No obstante, para ampliar la cobertura su empleo debe ser complementado con la trampa Malaise o algún otro artificio equivalente.

Palabras clave: desierto andino, cuencas andinas, ecosistemas andinos, comunidades de insectos, muestreo de insectos.

\begin{abstract}
The applicability of two methods for capturing and sampling hexapods inhabiting peatlands is communicated. The methods were based on the Barber and Malaise designs which were installed on three peatlands located in a High Andean sub-watershed of the Elqui River Basin (Region of Coquimbo, Chile). The study lasted three months and was conducted during the pick of biological activity at the site. In the analysis of taxonomic richness (Order in the case of Hexapoda and Family in the case of Diptera) were used the nonparametric estimators Chao 1, Chao-2, Jack-1, Jack-2 and Bootstrap. Together and for the entire study, the two combined methods provided 88217 specimens, recording a richness of 14 orders of Hexapoda and 29 families of Diptera. Both qualitatively and quantitatively the traps differed from each other in the record of taxa. At ordinal level, the combined methods gave a representation of $\sim 99 \%$ of the orders of Hexapoda present in the peatlands. In the case of Barber-trapping, this representation reached $~ 98 \%$; in the case of Malaise-trapping, it bordered $62 \%$. At level of families of Diptera, the combined methods gave a representation of $\sim 98 \%$. In turn, the representation with the Barber-trapping was $\sim 69 \%$, but skirted $84 \%$ with the Malaisetrapping. Since the Malaise trap was more affected by the windy conditions of the mountain than the Barber trap, the first one became a satisfactory complement to the latter, considering that the Barber trap not only caught soil animals, but also captured a significant fraction of flying and foliage-linked hexapods. The results of this work show that the Barber trap is a convenient device to study hexapods inhabiting these units of the Andean landscape. However, to expand coverage, its use must be complemented with Malaise trap or some other equivalent device.
\end{abstract}

Key words: andean desert, Andean watersheds, Andean ecosystems, insect communities, insect sampling.

1 Laboratorio de Entomología Ecológica. Departamento de Biología, Facultad de Ciencias, Universidad de La Serena, Casilla 599, La Serena, Chile.

2 Instituto de Entomología. Universidad Metropolitana de Ciencias de la Educación, Santiago-Chile.

3 Laboratorio de Ecología Animal. Departamento de Biología, Facultad de Ciencias, Universidad de La Serena, La Serena-Chile.

* E-mail: jcepeda@userena.cl

Fecha de Recepción: 21 Junio, 2013.

Fecha de Aceptación: 30 Septiembre, 2013. 


\section{Introducción}

Las vegas son elementos característicos del paisaje altoandino del norte-centro de Chile (Squeo et al., 2006a). Estas unidades proveen agua para el consumo humano y minero, constituyen reservorios y filtros de agua y sales, a la vez que son centros de biodiversidad del desierto andino y hábitat para importantes recursos biológicos (Squeo et al., 2006a; Cortés et al., 1995). Por estas razones, existe un creciente interés público y científico en establecer medidas de protección y mitigación, tendientes a asegurar su conservación y sustentabilidad ecológica (CONAF, 2003; Contreras 2002). Este logro requiere mejorar el conocimiento de la estructura y dinámica de estos ambientes.

Respecto de la biota de las vegas, la flora y vegetación está, en general, bien documentada (Osorio et al., 2006; Squeo et al., 2006a; 2006b; Ahumada y Faúndez, 2007); igualmente los elementos más carismáticos de la fauna de vertebrados (Cortés et al., 2006; Tabilo, 2006). Por el contrario, el conocimiento de la fauna de invertebrados de estos ecosistemas es más bien incipiente (Cepeda-Pizarro et al., 2006). Este trabajo se centró en el estudio del ensamble de artrópodos hexápodos asociados a la vegetación de tres vegas localizadas en una cuenca subsidiaria al río Elqui, en el norte-centro de Chile. Estos organismos cumplen diferentes funciones en el sistema de interés. Por ejemplo, son fragmentadores de materia orgánica, agentes polinizadores e importantes recursos tróficos de los vertebrados de la vega, particularmente aves (Squeo et al., 2006c; Tabilo, 2006). Los objetivos del estudio fueron a) evaluar y comparar la eficiencia de captura de las trampas Malaise y Barber, y b) evaluar y comparar las estimaciones de riqueza taxonómica obtenidas a base de la información proporcionada por estos métodos.

\section{Materiales y Métodos}

Las trampas fueron instaladas en tres vegas altoandinas ubicadas entre 3100 y $3800 \mathrm{~m} \mathrm{snm}$ (Figs. 1). Estas vegas forman parte de la subcuenca río del Toro, de la hoya hidrográfica del río Elqui (2945'S, 6959'O), en la Región de Coquimbo (Chile). Detalles del área de ubicación de las vegas se encuentran en Cepeda-Pizarro (2006). En este trabajo se evaluó la eficiencia de las trampas Malaise (TMA en adelante) y Barber (pitfall o de
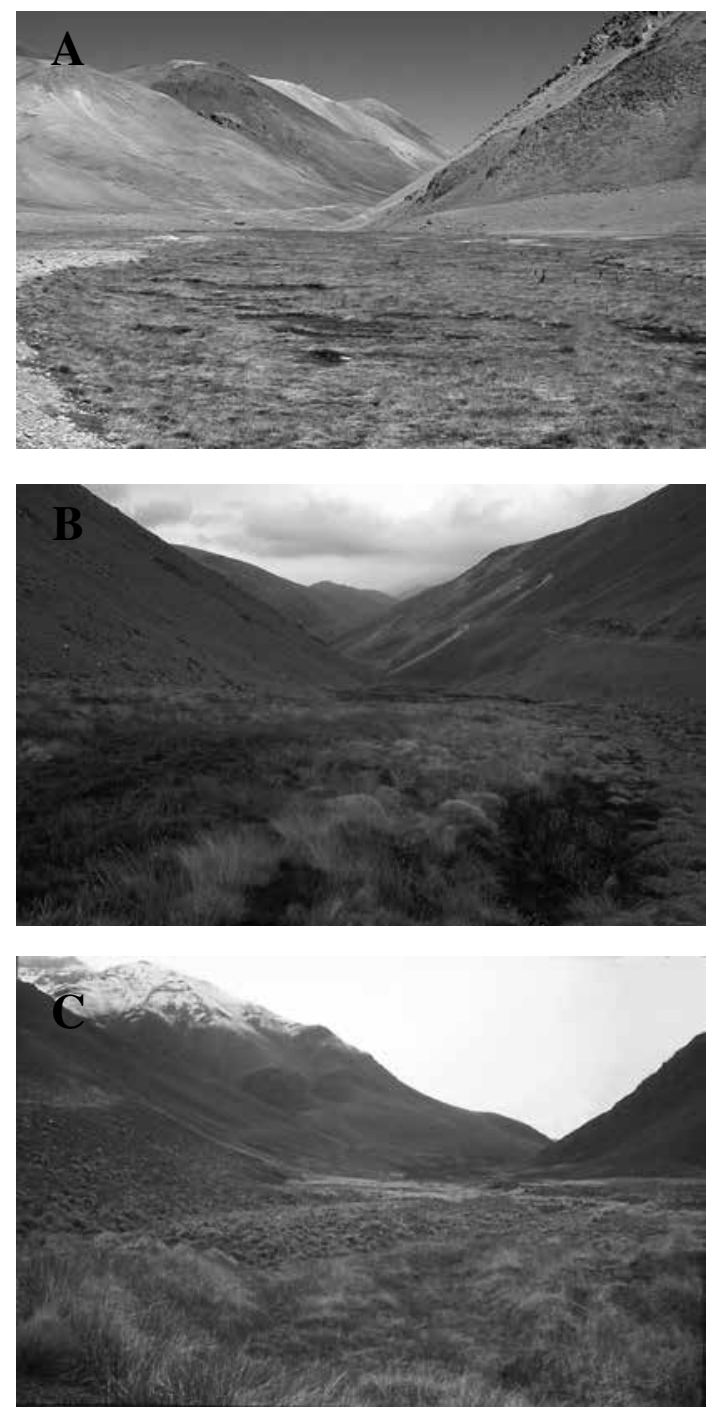

Fig. 1. Aspecto paisajístico de las vegas Tambo (A) (3800 n snm); Las Mulas (B) (3100 m snm) y Pastos Largos (C) (3660 m snm) de la subcuenca del río del Toro.

intercepción de caída) (TBA). El modelo usado de TMA tuvo dimensiones de $1,5 \mathrm{~m}^{2} \times 0,8 \mathrm{~m}$ de altura, de tela tul negro, incluyendo en su parte más alta un vaso colector de insectos conteniendo alcoholglicerinado $\left(70^{\circ}\right)$. El modelo de TBA correspondió al diseño descrito en Cepeda-Pizarro et al. (2005). Las trampas TBA se emplazaron en una parcela de 15 × 20 m ubicada en el centro de la vega. Se instalaron 20 TBA por vega, en disposición cuadricular, con separación de $5 \mathrm{~m}$ entre trampas. El vaso interior de TBA se llenó hasta dos tercios de su capacidad con una solución de formalina 
(5\%), glicerina y agua con detergente doméstico. Aledaño a la parcela de monitoreo se instaló una TMA. El trabajo de campo duró tres meses y fue realizado durante la estación de mayor actividad biológica del sistema (diciembre a marzo). Durante este período las trampas permanecieron activas quincenalmente, según la siguiente secuencia: del 1 al 15 diciembre (primer muestreo); del 9 al 24 de enero (segundo muestreo) y del 1 al 15 de febrero (tercer muestreo). Durante el receso, las TBA se inactivaron cubriendo su boca con un cuadrado de madera prensada, mientras que las TMA fueron retiradas y reacondicionadas. Los ejemplares capturados fueron removidos del contenedor letal, limpiados, secados y conservados en alcohol $\left(70^{\circ}\right)$ hasta el momento de su procesamiento y montaje. La identificación taxonómica de las capturas se realizó mediante comparación con material de referencia depositado en las colecciones del Museo Nacional de Historia Natural (Santiago, Chile), el Instituto de Entomología de la Universidad Metropolitana de Ciencias de la Educación (Santiago, Chile) y del laboratorio de Entomología Ecológica del Departamento de Biología de la Universidad de La Serena. Para la nomenclatura taxonómica ordinal se siguió a Artigas (1994) y de la Fuente (1994); para Diptera (Familias) se siguió a McAlpine et al. (1981, 1987). Duplicados del material capturado están depositados en la colección del Laboratorio de Entomología Ecológica (LEULS, Universidad de La Serena, La Serena, Chile).

La riqueza taxonómica del ensamble de Hexapoda (órdenes) y Diptera (Familias) se evaluó mediante los estimadores no paramétricos Chao1, Chao-2, Jack-1, Jack-2 y Boostrap, siguiendo a Colwell y Coddington (1994). Los cálculos se realizaron con el programa SDR-IV, con 50 aleatorizaciones (Seaby y Henderson, 2007). Para los efectos de las estimaciones, la serie vega $\mathrm{x}$ tiempo de muestreo fue considerada réplica.

\section{Resultados y Discusión}

\section{Relación de abundancias relativas}

Por todo el estudio y en conjunto se capturaron 88217 especímenes, el $74 \%$ fue aportado por TBA y el $\sim 26 \%$ por TMA (Tabla 1). Se registró la presencia de 14 órdenes. De estos, seis de ellos (i. e., Collembola, Microcoryphia, Dermaptera, Ephemeroptera, Orthoptera y Psocoptera) no fueron reportados por el muestreo-TMA (Tabla 1). Según las capturas-TBA, durante el período estudiado, el ensamble de hexápodos estuvo numéricamente dominado -considerando aquellos taxa con representación en la muestra de $\geq 3 \%$ total-TBA-,

Tabla 1. Órdenes de Hexapoda y número de individuos capturados por trampas Barber (TBA) y Malaise (TMA) instaladas en tres vegas altoandinas del norte-centro de Chile.

\begin{tabular}{|c|c|c|c|c|c|c|}
\hline \multirow{2}{*}{ Orden* } & \multicolumn{4}{|c|}{ Total/Tipo de trampa } & \multicolumn{2}{|c|}{ Total capturado } \\
\hline & TBA & $\%$ & TMA & $\%$ & $\mathrm{~N}^{\mathrm{o}}$ & $\%$ \\
\hline Coleoptera & 2.155 & 3,31 & 74 & 0,32 & 2.229 & 2,53 \\
\hline Collembola & 32.581 & 50,11 & 0 & 0,00 & 32.581 & 36,93 \\
\hline Dermaptera & 3 & $\leq 0,01$ & 0 & 0,00 & 3 & $\leq 0,01$ \\
\hline Diptera & 9.556 & 14,70 & 19.604 & 84,49 & 29.160 & 33,05 \\
\hline Ephemeroptera & 8 & 0,01 & 0 & 0,00 & 8 & 0,01 \\
\hline Hemiptera & 7.185 & 11,05 & 874 & 3,77 & 8.059 & 9,14 \\
\hline Hymenoptera & 2.702 & 4,16 & 772 & 3,33 & 3.474 & 3,94 \\
\hline Lepidoptera & 3.965 & 6,10 & 1.364 & 5,88 & 5.329 & 6,04 \\
\hline Microcoryphia & 1.610 & 2,48 & 0 & 0,00 & 1.610 & 1,83 \\
\hline Neuroptera & 32 & 0,05 & 18 & 0,08 & 50 & 0,06 \\
\hline Orthoptera & 534 & 0,82 & 0 & 0,00 & 534 & 0,61 \\
\hline Psocoptera & 9 & 0,01 & 0 & 0,00 & 9 & 0,01 \\
\hline Thysanoptera & 4.100 & 6,31 & 423 & 1,82 & 4.523 & 5,13 \\
\hline Trichoptera & 575 & 0,88 & 73 & 0,31 & 648 & 0,73 \\
\hline Total & 65.015 & 100,00 & 23.202 & 100,00 & 88.217 & 100,00 \\
\hline
\end{tabular}

* Órdenes están presentados alfabéticamente. 
por Collembola ( $\sim 50 \%)$, Diptera $(\sim 15 \%)$, Hemiptera $(\sim 11 \%)$, Thysanoptera y Lepidoptera $(\sim 6 \%)$, Hymenoptera $(\sim 4 \%)$ y Coleoptera $(\sim 3 \%)$. De acuerdo con el muestreo-TMA, el ensamble de hexápodos de las vegas estuvo numéricamente dominado por Diptera ( 84\%), Lepidoptera ( 6\%), Hemiptera ( 4\%) e Hymenoptera ( 3\%). Sumando los aportes de ambos métodos, la dominancia numérica correspondió a Collembola ( 37\%), Diptera ( 33\%), Hemiptera ( 9\%), Lepidoptera ( 6\%), Thysanoptera $(\sim 5 \%)$ e Hymenoptera ( 4\%) (Tabla 1$)$.

En atención al mejor conocimiento taxonómico de Diptera (nivel Familia) respecto de Collembola, se examinó con más detalles el ensamble del primero. Del total capturado (29160 especímenes), el muestreo-TMA aportó el $~ 69 \%$, contribuyendo con el registro de 25 Familias, en contraste con el registro de 20 Familias aportado por el trampeoTBA (Tabla 2). No obstante, hubo Familias cuya presencia no se registró con el muestreo-TMA (p. e., Asiilidae, Otitidae, Chloropidae, Nemestrinidae). Contrariamente, el muestreo-TBA no registró la presencia de Bibionidae, Culicidae, Psychodidae, Sarcophagidae, Simuliidae, Stratiomydae, Tachinidae y Therevidae. Según el muestreo-TBA, en el período estudiado, el ensamble de Familias de Diptera estuvo numéricamente dominado ( $\geq 3 \%$ totalTBA) por siete Familias: Chironomidae $(\sim 35 \%)$, Heleomyzidae ( 18\%), Ephydridae y Muscidae ( 10\%), Dolichopodidae ( 8\%), Syrphidae ( 6\%) y Phoridae $(\sim 4 \%)$. Según el muestreo-TMA, el ensamble estuvo numéricamente dominado por siete Familias: Muscidae ( 40\%), Sphaeroceridae ( 20\%), Chironomidae, Empididae y Heleomyzidae ( 7\%), Syrphidae $(\sim 5 \%)$ y Tachinidae ( $3 \%)$ (Tabla 2$)$. Combinando ambos métodos, las dominancias numéricas correspondieron a Muscidae $(\sim 31 \%)$, Chironomidae $(\sim 16 \%)$, Sphaeroceridae $(\sim 14 \%)$, Heleomyzidae ( $10 \%)$, Empididae $(\sim 6 \%)$, Syrphidae $(\sim 5 \%)$ y Ephydridae ( 4\%) (Tabla 2$)$.

\section{Riqueza taxonómica}

Considerando las capturas realizadas por ambos tipos de muestreo, los estimadores de riqueza taxonómica ordinal indicaron un rango de riqueza máxima de 13,8 a 14,3 (Tabla 3). En comparación a la riqueza observada $(\mathrm{S}=14)$, el promedio de los estimadores calculados sugiere una eficiencia de muestreo cercana al 98,5\%, lo que indica que, prácticamente, la mayoría de los órdenes representados en las vegas habrían sido capturados. Los estimadores calculados a base del muestreo Malaise entregaron valores más bajos que la riqueza observada. A su vez, los estimadores calculados basados en el muestreo Barber entregaron valores cercanos a la riqueza observada (Tabla 3 ). El promedio de los estimadores calculado según muestreo Malaise indicó una eficiencia de 62,4\% en comparación a la riqueza observada; por su parte, el trampeo Barber logró una eficiencia del 97,9\%. Estos datos muestran que las trampas Barber no solo capturaron elementos numéricamente dominantes asociados al suelo, sino que también colectaron grupos de hexápodos voladores y asociados al follaje, algunos de los cuales eran poco abundantes.

Respecto de las Familias de Diptera y sumando las capturas realizadas por ambos tipos de trampa, los estimadores de riqueza taxonómica indicaron un rango de riqueza máxima de 29,3 a 32,6 (Tabla 4). En comparación a la riqueza observada $(S=29$ Familias reconocidas), el promedio de los estimadores calculados sugiere una eficiencia de muestreo cercana al 97,5\%, lo que indica que, prácticamente, la mayoría de las Familias de Diptera representadas en las vegas, habrían sido capturadas. Con ambos tipos de trampa, los estimadores entregaron valores más bajos que la riqueza observada (Tabla 4). El promedio de los estimadores calculado según el muestreo Malaise indicó una eficiencia de 84,4\% en comparación a la riqueza observada; por su parte, el trampeo Barber logró una eficiencia del 69,3\%.

Existe una amplia gama de métodos de captura y muestreo de hexápodos asociados a ecosistemas terrestres (Southwood y Henderson, 2000). Cuando se seleccionan procedimientos de muestreo y captura para estudios de entomología de campo se debe tener presente la eficiencia del método en relación con el taxón o taxa de interés, las características del hábitat de los organismos, la forma en que estos están dispersos en él, la facilidad de instalación y manipulación de las trampas bajo las condiciones ambientales imperantes y el costo del programa de muestreo y captura, especialmente cuando se deben cubrir áreas relativamente extensas y atender los problemas de seudorreplicación. Considerando que la vegetación de las vegas estudiadas está formada por pastos bajos, en un ensayo exploratorio se probó la aplicabilidad de la red entomológica de rastreo. Esta, a pesar de ser ampliamente usada en el estudio de pastizales y cultivos bajos, no resultó adecuada en este ambiente. Las razones fueron 1) debido al 
Tabla 2. Familias de Diptera y números de especímenes capturados por trampas Barber (TBA) y Malaise (TMA) instaladas en tres vegas altoandinas del norte-centro de Chile.

\begin{tabular}{|c|c|c|c|c|c|c|}
\hline \multirow{2}{*}{ Familia } & \multicolumn{4}{|c|}{ Total/Tipo de trampa } & \multicolumn{2}{|c|}{ Total capturado } \\
\hline & TBA & $\%$ & TMA & $\%$ & $\mathrm{~N}^{\circ}$ & $\%$ \\
\hline Agromyzidae & 30 & 0,34 & 76 & 0,38 & 106 & 0,36 \\
\hline Asiilidae & 1 & 0,01 & 0 & $\leq 0,01$ & 1 & 0,00 \\
\hline Bibionidae & 0 & $\leq 0,01$ & 9 & 0,04 & 9 & 0,03 \\
\hline Bombyliidae & 172 & 1,92 & 168 & 0,83 & 340 & 1,17 \\
\hline Calliphoridae & 36 & 0,40 & 22 & 0,11 & 58 & 0,20 \\
\hline Ceratopogonidae & 10 & 0,11 & 168 & 0,83 & 178 & 0,61 \\
\hline Chironomidae & 3.134 & 35,07 & 1.431 & 7,08 & 4.565 & 15,66 \\
\hline Chloropidae & 198 & 2,22 & 0 & $\leq 0,01$ & 198 & 0,68 \\
\hline Culicidae & 0 & $\leq 0,01$ & 33 & 0,16 & 33 & 0,11 \\
\hline Dolichopodidae & 696 & 7,79 & 12 & 0,06 & 708 & 2,43 \\
\hline Empididae & 247 & 2,76 & 1.452 & 7,18 & 1.699 & 5,83 \\
\hline Ephydridae & 922 & 10,32 & 185 & 0,91 & 1.107 & 3,80 \\
\hline Heleomyzidae & 1.575 & 17,63 & 1.384 & 6,84 & 2.959 & 10,15 \\
\hline Muscidae & 944 & 10,56 & 8.184 & 40,47 & 9.128 & 31,30 \\
\hline Mycetophilidae & 51 & 0,57 & 62 & 0,31 & 113 & 0,39 \\
\hline Nemestrinidae & 1 & 0,01 & 0 & $\leq 0,01$ & 1 & 0,00 \\
\hline Otitidae & 12 & 0,13 & 0 & $\leq 0,01$ & 12 & 0,04 \\
\hline Phoridae & 349 & 3,91 & 226 & 1,12 & 575 & 1,97 \\
\hline Psychodidae & 0 & $\leq 0,01$ & 8 & 0,04 & 8 & 0,03 \\
\hline Sarcophagidae & 0 & $\leq 0,01$ & 237 & 1,17 & 237 & 0,81 \\
\hline Sciaridae & 0 & $\leq 0,01$ & 273 & 1,35 & 273 & 0,94 \\
\hline Simuliidae & 0 & $\leq 0,01$ & 280 & 1,38 & 280 & 0,96 \\
\hline Sphaeroceridae & 25 & 0,28 & 3.962 & 19,59 & 3.987 & 13,67 \\
\hline Stratiomydae & 0 & $\leq 0,01$ & 236 & 1,17 & 236 & 0,81 \\
\hline Syrphidae & 501 & 5,61 & 995 & 4,92 & 1.496 & 5,13 \\
\hline Tabanidae & 2 & 0,02 & 34 & 0,17 & 36 & 0,12 \\
\hline Tachinidae & 0 & $\leq 0,01$ & 697 & 3,45 & 697 & 2,39 \\
\hline Therevidae & 0 & $\leq 0,01$ & 60 & 0,30 & 60 & 0,21 \\
\hline Tipulidae & 9 & 0,10 & 30 & 0,15 & 39 & 0,13 \\
\hline Otros & 21 & 0,24 & 0 & $\leq 0,01$ & 21 & 0,07 \\
\hline Total & 8.936 & 100,00 & 20.224 & 100,00 & 29.160 & 100,00 \\
\hline
\end{tabular}

Tabla 3. Riqueza taxonómica observada (S) y estimada de órdenes de Hexapoda, mediante el empleo de trampas Barber (TBA) y Malaise (TMA).

\begin{tabular}{lcrc}
\hline Estimador & TBA & TMA & TBA+TMA \\
\hline Richness (S) & 14,0 & 8,0 & 14,0 \\
Chao-1 & 12,7 & 7,7 & 13,8 \\
Chao-2 & 13,3 & 7,6 & 14 \\
Jack-1 & 15,8 & 8,9 & 14,3 \\
Jack-2 & 15,1 & 10,8 & 13,9 \\
Bootstrap & 14,6 & 8,7 & 14,2 \\
\hline
\end{tabular}

nivel altitudinal de las vegas (3100-3800 m snm), el esfuerzo físico resultó demasiado agotador, incluso para personal experimentado; 2) las vegas son espacios abiertos ventosos, con rachas entre 34
Tabla 4. Riqueza taxonómica observada (S) y estimada de Familias de Diptera, mediante el uso de trampas Barber (TBA) y Malaise (TMA).

\begin{tabular}{lccc}
\hline Estimador & TBA & TMA & TBA+TMA \\
\hline Richness (S) & 21,0 & 25,0 & 30,0 \\
Chao-1 & 16,3 & 22,8 & 29,4 \\
Chao-2 & 18,2 & 21,9 & 29,3 \\
Jack-1 & 23,4 & 27,8 & 32,6 \\
Jack-2 & 25,3 & 28,2 & 31,5 \\
Bootstrap & 20,8 & 25,9 & 31,1 \\
\hline
\end{tabular}

a $72 \mathrm{~ms}^{-1}$, lo que dificultó el desplazamiento del operador, el traspaso de los especímenes al frasco colector y el funcionamiento de la bolsa colectora; 3 ) en virtud de que la temperatura del aire baja 
rápidamente pasado mediodía, sobre todo bajo condiciones ventosas, la sensación térmica obligó a usar ropas gruesas, dificultando el desplazamiento y el manejo de la red; 4) la superficie de la vega no es pareja, conteniendo sitios anegados o fangosos, lo que dificultó el desplazamiento según el procedimiento de muestreo establecido, y 5) la vegetación es muy achaparrada, pegada al sustrato y dura, por lo que la amplitud del movimiento de rastreo no se pudo mantener constante.

Los otros dos métodos evaluados son ampliamente usados. Aun cuando el diseño Malaise constituye un artificio adecuado para el muestreo y captura de insectos voladores, no todos los taxa presentes en las vegas estuvieron adecuadamente representados en las muestras. El diseño Barber es frecuentemente usado en entomología de campo. Enterradas en el sustrato, las trampas Barber se recomiendan especialmente para ambientes con estructura vegetacional simple. Su eficiencia no solo depende de la abundancia de los individuos de interés, sino también de su vagilidad y los números que entregan corresponden a un concepto definido como "densoactividad" (Saska et al., 2013). Por lo mismo, su eficiencia depende de factores que se interponen en el libre desplazamiento o que alteran la conducta habitual de los individuos (p. e., viento, temperatura, luminosidad lunar). No obstante, tiene a su favor su bajo costo, su facilidad de instalación, su independencia respecto de un operador y su replicabilidad. A pesar de que el diseño Barber no es el más adecuado para insectos voladores (Work et al., 2002), en el presente estudio este dio cuenta satisfactoria no solo de los hexápodos asociados al suelo, sino también de aquellos presentes en el follaje y de formas voladoras. La presencia porcentual significativa de Diptera registrada en este trabajo concuerda con resultados similares hallados en trabajos previos realizados en sitios costeros del desierto transicional de Chile (Cepeda-Pizarro et al., 2005) y en otras latitudes (Blades y Marshall, 1994; Acosta y Prat, 2010). Comparando ambos diseños, los intensos vientos imperantes en el área de estudio probablemente ejercieron un efecto negativo mayor sobre la efectividad de captura del diseño Malaise que sobre el diseño Barber. En efecto, aun cuando las trampas Malaise fueron confeccionadas teniendo presente esta característica del entorno, ellas debieron quedar, durante su funcionamiento, firmemente sujetas al sustrato y luego ser reacondicionadas en los períodos intermuestrales; en el caso del diseño
Barber, la trampa, al estar enterrada, habría quedado protegida por las alteraciones microtopográficas de la superficie de la vegetación y por su forma acolchonada. Finalmente, los resultados de este estudio muestran que trabajos orientados al estudio de los ensambles de Hexapoda en ambientes andinos debieran considerar, por sus bondades, simultáneamente ambos métodos de captura y muestreo.

\section{Conclusiones}

Algunas Familias de Diptera fueron adecuadamente registradas por las trampas Malaise. No resultaron satisfactorias para otros grupos de hexápodos voladores (p. e., Trichoptera). Los intensos vientos imperantes habrían afectado la eficiencia de este diseño de trampas. Este efecto habría sido menor en el caso de las trampas Barber por encontrarse enterradas a nivel de la superficie de la vegetación y protegida por esta. Comparadas con las trampas Malaise, las trampas Barber no solo fueron eficientes en la captura de hexápodos asociados al suelo, sino también lo fueron con algunos grupos de hexápodos voladores o asociados a la vegetación. Aunque con sesgo en contra de Diptera, estas trampas capturaron una muestra más diversa de órdenes que las trampas Malaise.

A nivel ordinal, el muestreo combinado entregó una representación del $\sim 99 \%$ de los órdenes de Hexapoda presentes en las vegas. En el caso del trampeo Barber, esta representación alcanzó el 98\%. En el caso del trampeo Malaise, esta representación bordeó el 62\%. A nivel de Familias de Diptera, el muestreo combinado entregó una representación del $\sim 98 \%$. En el caso del trampeo Barber, esta representación alcanzó al $\sim 69 \%$. En el caso del trampeo Malaise, esta representación bordeó el $84 \%$.

De lo anterior se concluye que trabajos orientados al estudio de los ensambles de Hexapoda en estos ambientes debieran considerar simultáneamente ambos métodos de captura y muestreo.

\section{Agradecimientos}

Este estudio fue financiado por la Dirección de Investigación de la Universidad de La Serena (Convenio de Desempeño en Investigación, JCP), DIULS PR13121 y VACDDI001 (JPA). Los autores agradecen a J. Rau A. por sus sugerencias al análisis de riqueza taxonómica. 


\section{Literatura Citada}

Acosta, O.; Custodio, E.

2008. Impactos ambientales de las extracciones de agua subterránea en el salar de Huayco (norte de Chile). Boletín Geológico Minero, 119: 33-50.

Acosta, R.; Prat, N.

2010. Chironomid assemblages in high altitude streams of theAndean region of Peru. Fundamental and Applied Limnology, 177: 57-79.

Ahumada, M.; Faúndez, L.

2007. Manual de reconocimiento de especies de flora de las veranadas: Región de Coquimbo. Servicio Agrícola y Ganadero, División de Protección de Recursos Naturales Renovables, Coquimbo, 146 pp.

Artigas, J. N.

1994. Entomología económica. Insectos de interés agrícola, forestal, médico y veterinario (Nativos, introducidos y susceptibles de ser introducidos). Volúmenes I y II, Ediciones Universidad de Concepción, Concepción, Chile, 1126 pp., $942 \mathrm{pp}$.

Blades, D.C.A.; Marshall, S.A.

1994. Terrestrial arthropods of Canadian peatlands - synopsis of pan trap collections at 4 Southern Ontario Peatlands. Memoirs of the Entomological Society of Canada 169: 221-284.

Cepeda-Pizarro, J. (ed.).

2006. Geoecología de los Andes Desérticos: La Alta Montaña del Valle del Elqui. Ediciones Universidad de La Serena. Universidad de La Serena, La Serena-Chile, 551 pp.

Cepeda-Pizarro, J.; Pola, M.; Zuleta, C.; González, C.

2006. Relaciones de abundancia y diversidad de la entomofauna del humedal Tambo-Puquíos. En: Geoecología de los Andes Desérticos: La Alta Montaña del Valle del Elqui. Ediciones Universidad de La Serena. Universidad de La Serena, La Serena-Chile, pp. 475-521.

Cepeda-Pizarro, J.; Pizarro-Araya, J.; Vásquez, H.

2005. Variación en la abundancia de Arthropoda en un transecto latitudinal del desierto costero transicional de Chile, con énfasis en los tenebriónidos epígeos. Revista Chilena de Historia Natural, 78: 651-663.

Colwell, R.K.; Coddington, J.A.

1994. Estimating terrestrial biodiversity through extrapolation. Philosophical Transaction of the Royal Society of London, 345: 101-118.

CONAF.

2003. Plan de acción para la conservación sustentable y uso racional de humedales altoandinos. Corporación Forestal (Ministerio de Agricultura)-Convención Ramsar. SantiagoChile, 23 pp.

Contreras, J.P.

2002. Norte de Chile: conservación de humedales altoandinos para un desarrollo productivo sustentable. Revista Ambiente y Desarrollo, XVIII: 125-131.

Cortés, A.; Torres-Mura, J.C.; Contreras, L.; Pino, C. 1995. Fauna de Vertebrados de los Andes de Coquimbo: Cordillera de Doña Ana. Ediciones Universidad de La Serena. Universidad de La Serena, La Serena-Chile, 96 pp.

Cortes, A.; Miranda, E.; López-Cortés, F.

2006. Abundancia y dieta del camélido Lama guanicoe en un ambiente altoandino del norte-centro de Chile. En: Geoecología de los Andes Desérticos: La Alta Montaña del Valle del Elqui. Ediciones Universidad de La Serena. Universidad de La Serena, La Serena-Chile, pp. 383-411.

De la Fuente, J.A.

1994. Zoología de artrópodos. Interamericana McGraw-Hill Nueva York-USA, 805 pp.

McAlpine, J.F.; Peterson, B.V.; Shewell, G.E.; Teskey, H.J.; Vockeroth, J.R.; Wood, D.M. (eds.).

1981. Manual of Neartic Diptera. Monograph 27 (Vol. 1). Research Branch, Agriculture, Canada. Ottawa-Canada $674 \mathrm{pp}$.

McAlpine, J.F.; Peterson, B.V.; Shewell, G.E.; Teskey, H.J.; Vockeroth, J.R.; Wood, D.M. (eds.).

1987. Manual of Neartic Diptera. Monograph 28 (Vol. 2). Research Branch, Agriculture, Canada. Ottawa-Canada, $1332 \mathrm{pp}$.

Osorio, R.; Cabezas, R.; Reyes, H.; Álvarez, P.; Koné, T.

2006. Humedales altoandinos de la IV Región. En:

Geoecología de los Andes desérticos. La Alta Montaña del Valle del Elqui. Cepeda-Pizarro, J. (ed.). Ediciones Universidad de La Serena. Universidad de La Serena, La Serena-Chile, 152-239.

Saska, P., Van der Werf, W., Hemerik, L., Luff, M.L., Hatten, T.D.; Honek, A.

2013. Temperature effect on pitfall catches of epigeal arthropods: a model and method for bias correction. Journal of Applied Ecology, 50: 181-189.

Seaby, R.M.H.; Henderson, P.A.

2007. Species Diversity and Richness-IV: Measuring and understanding biodiversity. Piscis Conservation Ltd. Lymington, Hampshire-UK, 84 pp.

Southwood, T.R.E.; Henderson, P.A.

2000. Ecological Methods. Blackwell Science, Malden MAUSA, 575 pp.

Squeo F.A.; Warner, B.G.; Aravena, R.; Espinoza, D. 2006a. Bofedales: high altitude peatlands of the central Andes. Revista Chilena de Historia Natural, 79: 245-255.

Squeo, F., Cepeda-Pizarro, J.; Olivares, N.; Arroyo, M.T.K. 2006b. Interacciones ecológicas en la alta montaña del valle del Elqui. En: Geoecología de los Andes desérticos. La Alta Montaña del Valle del Elqui. Cepeda-Pizarro, J. (ed.). Ediciones Universidad de La Serena. Universidad de La Serena, La Serena, Chile, pp. 69-103.

Squeo, F.A.; Ibacache, E.; Wagner, B.; Espinoza, E.; Aravena, R.; Gutiérrez, J.G.

2006c. Productividad y diversidad florística de la vega Tambo, Cordillera de Doña Ana. En: Geoecología de los Andes desérticos. La Alta Montaña del Valle del Elqui. CepedaPizarro, J. (ed.). Ediciones Universidad de La Serena. Universidad de La Serena, La Serena-Chile, 325-351.

Tabilo, E. Avifauna del humedal Tambo-Puquíos.

2006. En: Geoecología de los Andes desérticos. La Alta Montaña del Valle del Elqui. Cepeda-Pizarro, J. (ed.). Ediciones Universidad de La Serena. Universidad de La Serena, La Serena-Chile, 355-379.

Work, T.T.; Buddle, C. M.; Korinus, L.M.; Spence, J.R.

2002. Pitfall trap size and capture of three taxa of litterdwelling arthropods: Implications for biodiversity studies. Environmental Entomology, 31: 438-448. 
